\title{
Construction method of enterprise-level database operational measurement platform based on bidirectional coupling algorithm
}

\author{
Hongwen Xia, \\ College of Teacher Education, Zhejiang Normal University, \\ Jinhua, 321004 \\ China
}

Received: June 29, 2021. Revised: December 17, 2021. Accepted: January 2, 2022. Published: January 3, 2022.

\begin{abstract}
When identifying the enterprise database data information, the enterprise database operational dimension measurement platform based on the bilinear method will produce ringing or overshoot effect, the database data monitoring effect is poor as well as the accuracy of operation measurement. To solve this problem, this paper proposes a method to build an enterprise level database operation and maintenance measurement platform based on bidirectional coupling algorithm. Build enterprise database operation measurement platform. The enterprise database monitoring platform is connected with the monitoring database by remote database chain. The Oracle database job scheduling method is used to obtain the monitoring index information in the monitored database, and the database memory performance is comprehensively evaluated by MPI (Memory Perform Index). The platform uses semantic capture layer and related analysis layer to distinguish user behavior, analyze user experience satisfaction, and realize the operation measurement of enterprise level database. The experimental results show that the operational measurement platform built by this method has high throughput, low memory occupancy rate, high measurement accuracy and good user experience.
\end{abstract}

Keywords-Bidirectional coupling algorithm, enterprise-level database, monitoring indicator information, operation measurement, platform, user behavior.

\section{INTRODUCTION}

$\mathrm{W}$ ITH the integration of the global economy and the advent of the internet information era, the data is increased gradually, and the database technology is constantly improving, especially in the process of enterprise-level information construction, the database has become a required item for enterprise informatization [1] [2]. Oracle databases are increasingly recognized by enterprises and can periodically collect information of various databases monitoring index and save it to enterprise-level databases. The operation measurement of enterprise database is of great application value to ensure the smooth operation of the database and improve the satisfaction of the database user. However, there are some problems in the process of enterprise database operation and maintenance, such as operation hidden dangers, high complexity of management technology, inconsistent operation and maintenance methods, lack of daily inspection and monitoring, low efficiency and so on.

The enterprise-level database operation measurement platform, which is traditionally constructed by bilinear method, will have ringing or overshoot effect when identifying the enterprise database data information, and does not have the function of enterprise database user behavior and user satisfaction analysis, so it makes the accuracy of database data monitoring and operation measurement reduce greatly. However, the throughput of the database operation measurement platform is low, so it cannot achieve high-precision measurement and monitoring; There is also a database operational measurement analysis platform based on adaptive baseline, build a loosely coupled hierarchical logical architecture, realize the active management and trend prediction of the database based on the adaptive baseline, establish the measurement and analysis method based on the adaptive baseline, realize the low-cost and efficient database operation and maintenance mode, and enhance the stability of database operation, which realizes the active, preventive database operation mode and the trend management of database, greatly reducing the hidden problems in database operation, but there are the disadvantages of large computation and high energy consumption.

In view of the disadvantages of traditional platform construction methods, this paper proposes the enterprise-level database operational measurement platform construction method based on the bidirectional coupling algorithm, 
constructs the two-way coupling diffusion model of information in the enterprise database through the two-way coupling algorithm, and identifies the relationship between the raw data, diffusion coefficient and time step in the data information. And based on this, an enterprise-level database operational measurement platform is built [3]. The experimental results show that the platform has high throughput rate, small occupy memory, stable operating condition, and achieves high-precision measurement and monitoring of enterprise-level database data [4].

\section{CONSTRUCTION Method OF ENTERPRISE-LEVEL DAtabase OPERATIONAL MEASUREMENT PlatFoRM}

\section{A. Bidirectional Coupling Algorithm}

(1) Unified bidirectional diffusion framework

Partial Differential Equation (PDE) has developed rapidly in recent years in identifying data information of enterprise-level databases [5]. Anisotropic diffusion filter (also known as Perona Malik filter) is mainly used to smooth the image, which overcomes the defect of Gaussian filter blur. Anisotropic diffusion can smooth the image while preserving the image edge; In addition, Perona Malik filter has been applied in geological image processing, such as smoothing DEM data, channel extraction and so on. Non-linear anisotropic diffusion filters are commonly used in data denoising. The data processed by this filter has good accuracy and high maturity. The data $o(x, y, t): \Omega \times[0, \infty) \rightarrow T$ is diffused by the following equation:

$$
\frac{\beta o(x, y, t)}{\beta t}=\operatorname{div}((g|\nabla o(x, y, t)|) \nabla o(x, y, t))
$$

In the equation, $g(|\nabla o|)$ is scalar diffusion coefficient, and the diffusion coefficient is determined as a non-increasing function that regulates the behavior of the diffusion equation.

Anisotropic Diffusion with Shock Filter (ADSF) is proposed by L. Mazorra and L. Alvarez, this filter connect shock filter and anisotropic diffusion filter proposed by Rudin and Osher, moreover, the filter is also a data enhancement method based on partial differential equation, and the enhancement expression is as follows:

$$
\frac{\beta o}{\beta t}=-\operatorname{sign}\left(J_{\tau} o_{N N}\right)|\nabla o|+d o_{T T}
$$

Of which, $\operatorname{sign}(\cdot)$ and $J_{\tau}$ are sign functions and Gaussian function whose standard deviation is $\tau$ respectively; $d$ is a constant and also a positive number; $o_{N N}$ is the second-order direction derivative in the gradient direction, $o_{T T}$ is the second-order direction derivative in the tangent direction.

According to the nature of sign function $\operatorname{sign}(y)$, the following equation is obtained:

$$
\operatorname{sign}(y)=\frac{y}{|y|}, y \neq 0
$$

Therefore, a uniform bidirectional diffusion formula (BDF) including (1) and (2) is obtained, it is shown as follows:

$$
\begin{gathered}
\frac{\beta o}{\beta t}=\lambda\left(-d_{N}\left(o_{N}, \mathrm{o}_{\mathrm{NN}}, \mathrm{o}_{\mathrm{TT}}\right) \mathrm{o}_{\mathrm{NN}}\right)+ \\
\eta\left(\mathrm{d}_{\mathrm{T}}\left(o_{N}, \mathrm{o}_{\mathrm{NN}}, \mathrm{o}_{\mathrm{TT}}\right) \mathrm{o}_{\mathrm{NN}}\right)
\end{gathered}
$$

In the formula, $\eta$ and $\lambda$ are the forward and reverse diffusion control coefficients respectively, $d_{N}(\cdot)$ and $\mathrm{d}_{\mathrm{T}}(\cdot)$ are diffusion coefficients which parameters are $\left(o_{N}, \mathrm{o}_{\mathrm{NN}}, \mathrm{o}_{\mathrm{TT}}\right)$.

(2) Bidirectional coupled diffusion model

In (2), judging the data by zero-crossing of the second-order directional derivative is a binary decision-making process, which recognizes the data in the enterprise-level database as non-numeric data. In order to improve the accuracy of enterprise-level database data recognition, hyperbolic tangent function $\operatorname{th}(\cdot)$ is used to replace the sign function $\operatorname{sign}(\cdot)$.

If (4) is realized after iteration, the forward force and the backward force in the equation will cancel, and then (4) is divided into two formulas, getting the Coupled bidirectional flow (CBDF) model. The model iterates over time, and the formula is:

$$
\left\{\begin{array}{l}
w^{0}=o^{0} \\
w^{n+1}=o^{n}+\Delta t L_{N}^{n}\left(o^{n}\right), L_{N}(\pi)=\lambda\left(-d_{N} t h\left(l o_{N N}\right)\right) \\
O^{n+1}=o^{n+1}+\Delta t L_{T}^{n}\left(w^{n+1}\right), L_{T}(w)=\eta\left(d_{T} w_{T T}\right)
\end{array}\right.
$$

In formula, $o^{0}$ and $\Delta t$ are the raw data ( $w$ is an intermediate variable) and time step ( $n$ is the number of iterations) respectively, other parameters have the same meaning as (4).

$o^{0} \rightarrow w^{0} \rightarrow w^{1} \rightarrow o^{1} \rightarrow w^{2} \rightarrow o^{2} \rightarrow \cdots$ its data sequence, and the sequence is iteratively computed to obtain the recognition result.

In order to maintain data characteristics in the process of data recognition, how to select diffusion coefficient $d_{N}(\cdot)$ and $d_{T}(\cdot)$ is discussed as follows. In the past enterprise database data information identification process, data will produce ringing or overshoot effect. The diffusion speed $d_{N}$ of enterprise database information is limited only by the gradient information. When the number of iterations increasing, the data information overshoot point in the enterprise-level database increases gradually, and the speed is faster and faster, producing reverse diffusion phenomenon [6] [7]. Therefore, in order to control the directional diffusion of information in the enterprise-level database, the second derivative information is added and the following formula is obtained:

$$
\begin{aligned}
& d_{N}=\left|o_{N}\right| /\left(1+l_{1} o_{N N}^{2}\right) \\
& d_{T}=1 /\left(1+l_{2} o_{T T}^{2}\right)
\end{aligned}
$$

Through the above series of equations to obtain the bidirectional coupled diffusion model of information in enterprise-level database, the model can identify the original data, diffusion coefficient and the relationship between time step of enterprise-level database data information, and build the 
enterprise-level database operational measurement platform on that basis [8].

\section{B. Construction of Enterprise-Level Database Operational Measurement Platform}

(1) The general design idea

The method of establishing remote database links is used to connect the enterprise-level database monitoring platform and different monitored databases. The database job scheduling method is used in the monitored database to obtain all monitoring index information, and the monitoring index information is stored in the database monitoring server database. In Fig. 1, the enterprise-level database operation measurement platform is constructed.

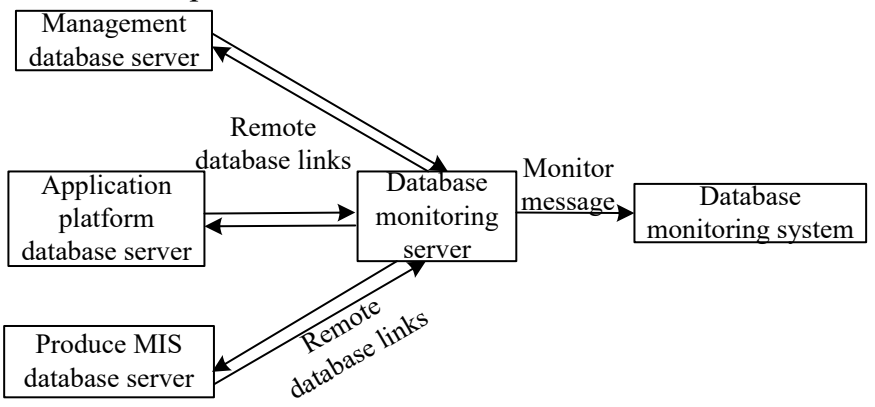

(2) Platform features

Fig. 1 The structure of platform

Establish remote database links: The enterprise database monitoring platform is connected to many monitored databases, and by the method of building the remote database links, the test data is entered into different monitored database platforms to obtain the corresponding monitoring information [9]. If a remote database connection is often used, building the remote database links to specify the remote database connection username and connection descriptor [10]. Assuming that any SQL statement specifies remote database links, then the remote database links pop up a dialog box and fulfill the SQL statement, and finally return to the database.

The enterprise information acquisition database operational measurement platform obtains the monitoring index information in the monitored database through the database job scheduling on a regular basis, and this process is carried out in a circular way [11]. The process of collecting monitoring indicator information is automatically implemented through the Oracle database job scheduling method [12].

The time and actions to perform an Oracle database job are determined in advance. Actions performed include SQL statement SL/SQL blocks, JAVA stored procedures, PL/SQL stored procedures, external procedures, and all executable files stored on the server file platform (all scripts and binary executables are available).

The start time of the job is controlled by the execution time of the action, and the time to execute the action is taken as the cycle time interval of the operation [13] [14].

Through the Memory Perform Index (MPI), the memory performance of enterprise-level database is evaluated. The enterprise-level database operational measurement platform uses MPI as the indicator of the situation of database memory usage and allocation, and meanwhile evaluates the database memory performance through MPI comprehensively, and obtains a performance score, which is used to determine the database operation [15].

Table I The indexes of MPI

\begin{tabular}{ccc}
\hline Classification & Required Level & Top score \\
\hline The hit rate of buffer cache & $>98 \%$ & 30 \\
The hit rate of dictionary cache & $>98 \%$ & 30 \\
The hit rate of library cache & $>98 \%$ & 30 \\
The percentage of sort in memory of the total sort & $>98 \%$ & 30 \\
MPI index & $<5 \%$ & 30 \\
The percentage of the top 10 statements that waste the most memory of total memory reads & Total score & 150 \\
\hline
\end{tabular}

The platform of this paper is divided into two layers, namely the semantic capture layer and the association analysis layer. The output, input, and interrelationships of the semantic capture layer and the association analysis layer are shown in Fig. 2.

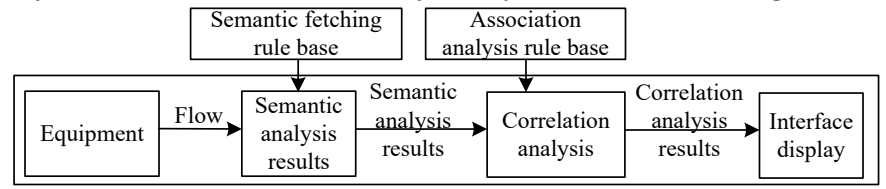

Fig. 2 The design of platform layered architecture

As can be seen from Fig. 2, the platform matches the flow and semantic fetching layer rule base used by the user through the semantic fetching layer, and obtains the output that is the response of different requests to all users. The input of the correlation analysis layer is the output obtained through the semantic fetching layer, and the correlation analysis layer connect and analyze the input data information and the association analysis rule base provided by the lower layer, and the user behavior is judged by the above analysis, and the user's experience satisfaction is analyzed according to all user behavior.

\section{EXPERIMENTAL ANALYSES}

\section{A. Throughput Test}

The experimental test adopts the method of this paper for the throughput rate of financial database operation measurement platform established by a logistics company's financial management. On the platform, the platform throughput is tested using offline flow, namely, to handle mixed flow of different sizes and proportions, and to test the throughput of the platform according to the time required to process all offline flow. Set the target flow of this platform as the flow of the financial management and control system of the logistics enterprise, and mix pure target flow and other unrelated flows in different 
proportions. As shown in Table II, test the time required for this platform to process these flows, which is the platform throughput. The throughput platform interface is shown in Fig. 3 .

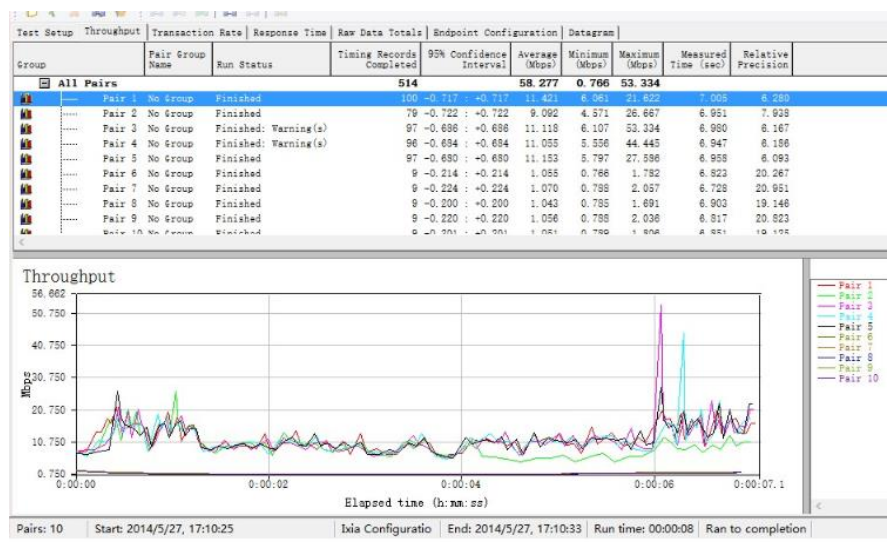

Fig. 3 Throughput platform interface

Throughput of the platform under different mixed flows in Fig. 4.

Table II Different proportions of mixed flow

\begin{tabular}{|c|c|c|c|c|c|}
\hline Name & Flow 1 & Flow 2 & Flow 3 & Flow 4 & Flow 5 \\
\hline Size /byte & 8759677 & 12147937 & 161157625 & 25996896 & 74728217 \\
\hline the proportion of target flow & $91.73 \%$ & $72.13 \%$ & $53.21 \%$ & $32.61 \%$ & $11.76 \%$ \\
\hline Average grouping length /byte & 685.9 & 695.9 & 696.3 & 736.9 & 771.1 \\
\hline \multirow{6}{*}{ 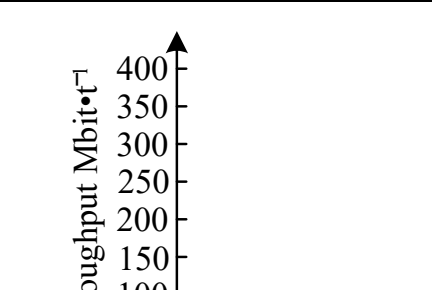 } & & & 36.15 & 23.82 & 21.15 \\
\hline & & & 38.42 & 21.25 & 19.98 \\
\hline & & & 40.09 & 23.94 & 20.13 \\
\hline & & & 39.88 & 24.95 & 21.56 \\
\hline & & & 35.97 & 22.03 & 20.88 \\
\hline & & & 40.12 & 25.16 & 23.00 \\
\hline
\end{tabular}

According to Table III, the maximum peak signal-to-noise ratio obtained by this method is 40.12 , the maximum peak signal-to-noise ratio obtained by bicubic method is 25.12 , and the maximum peak signal-to-noise ratio obtained by bilinear method is 23.0. Compared with the bilinear method and bicubic method, the average peak signal-to-noise ratio obtained by this method is the largest, which indicates that the data noise in enterprise database is smaller after using this method, so it can identify the data information in the enterprise database more accurately, and provide help for the operation measurement of the enterprise database.

\section{B. Measurement Accuracy Analysis}

The experiment compares the accuracy of the above 6 data

\section{A. The Peak Signal-to-Noise Ratio}

The experiment uses the method in this paper, bilinear method and bicubic method to identify six kinds of data information in the experimental logistics enterprise database, and calculate the peak signal-to-noise ratio PSNR of the identification information of different methods. The comparison results are shown in Table III.

Table III PSNR comparison of different identification methods

\begin{tabular}{|c|c|c|c|}
\hline Data type & $\begin{array}{l}\text { Method in } \\
\text { this paper }\end{array}$ & $\begin{array}{l}\text { Bicubic } \\
\text { method }\end{array}$ & $\begin{array}{l}\text { Bilinear } \\
\text { method }\end{array}$ \\
\hline
\end{tabular}


Fig. 5 Data measurement comparison results of different methods

According to Fig. 5, in the process of measuring enterprise-level database data information, compared with the bilinear method and bicubic method, the method in this paper has the highest accuracy, the accuracy is maintained at about $98 \%$, while the average data measurement accuracy of the bicubic method is about $86 \%$, and the average data measurement accuracy of the bilinear method is only about $77 \%$. The accuracy of the method in this paper measuring 6 kinds of data is in a stable state, while the other two methods fluctuate greatly, so the experiment shows that the accuracy of method in this paper measuring the enterprise-level database data information is high. This is because this method uses the nonlinear anisotropic diffusion filter for data denoising, and the data processed by this filter has high accuracy and high maturity. The hyperbolic tangent function is used to replace the sign function to improve the accuracy of data recognition in enterprise database.

\section{Platform Stability Analysis}

To verify the effectiveness of enterprise database operation metrics, the experiment imports the target flow from other devices into the platform of this paper, and test the platform device for a long time to simulate the online situation, test the platform memory usage and platform stability of this paper. Fig. 6 illustrates the amount of memory required for the various routines during the 48-hour run of the platform in this paper.

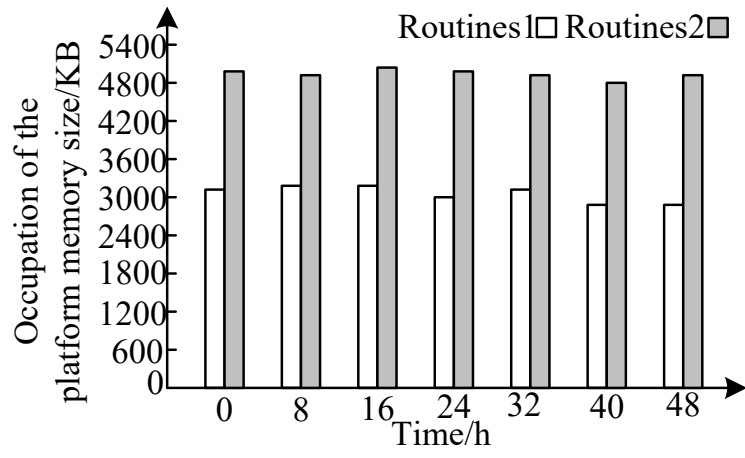

Fig. 6 Memory usage of the platform running for 48 hours

As shown in Fig. 6, with the increase of time, during the 48-hour run of platform in this paper, the size of the memory occupied by routine 1 and routine 2 has not changed much. The memory value of routine 1 is floating around $3000 \mathrm{~KB}$, while the memory value of routine 2 is floating around $5000 \mathrm{~KB}$, which indicates that the memory cost of this paper is stable, and the platform is stable in operation. So it helps to improve the effectiveness of the enterprise-level database operational measurement. This is because this method increases the second derivative information to control the directional diffusion of information in enterprise database. The platform obtains the monitoring index information in the monitored database regularly through the database job scheduling and circulates the implementation.

\section{The User Experience Time Analysis}

Test the experience time of users' using this platform for financial analysis, and the result is shown in Fig. 7.

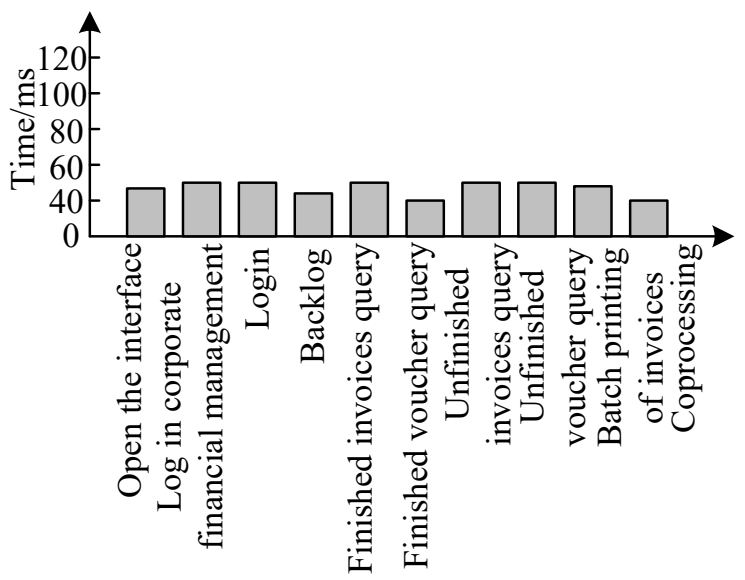

Fig. 7 User experience time statistics

According to Fig. 7, in the process of financial analysis by the user using this platform, the time taken for each operation is less than $50 \mathrm{~ms}$, and the time changes are relatively stable without obvious fluctuations, which indicates that the user has high efficiency in financial analysis through the platform and has excellent user experience. The main purpose of this paper is to match the flow used by users through the semantic acquisition layer and the rule base of the semantic acquisition layer, and obtain the output as the response to different requests of all users, judge the user behavior through the above analysis, and analyze the user experience satisfaction according to all user behaviors.

\section{CONCLUSIONS}

The advantages of the enterprise-level database operation measurement platform based on the bidirectional coupling algorithm in this paper are as follows:

(1) The platform constructs the two-way coupling diffusion model of enterprise database through the bidirectional coupling algorithm, which effectively identifies the relationship between the raw data, diffusion coefficient and time step in the enterprise database data information;

(2) The platform monitors the daily working status of the enterprise-level database, statistics the database information, and diagnoses and analyzes the problem information in the database, finds the problem and solves the problem in time, and ensures the continuous operation of the enterprise business; The platform adopts Oracle database job scheduling method to obtain the monitoring index information in the monitored database, evaluate database memory performance through MPI comprehensively, and provide a reliable basis for database scheduling;

(3) The platform uses semantic fetching layer and correlation analysis layer to judge user's behavior and analyzes user's experience satisfaction, which can reduce the coupling between the database modules in the platform, ensure that testing and development, developments, development and testing do not interfere with each other, and enhance the efficiency of enterprise business development effectively. 
(4) In the future development, we should supplement the experimental test indicators, increase the accuracy of the test results, and provide enterprise business development efficiency.

\section{References}

[1] Z. Chen, and Y. Yang, "The establishment and application of the geophysical spatial correlation algorithm of the coupling scale," Journal of Geo-Information Science, vol. 20 , no. 1 , pp. $37-47,2018$

[2] W. Li, G. Xu, and H. Wei, "Research on the integrated corridor operation and maintenance management system based on BIM," Journal of Underground Space and Engineering, vol. 14, no. 2, pp. 287-292, 2018.

[3] H. Lai, X. Deng, and H. Chen, "Urban rail transit operation and maintenance model delivery standard based on BIM," Urban Rapid Rail Transit, vol. 28, no. 3, pp. 78-83, 2015.

[4] L. Wang, Z. Qian, J. Guo, "Ocean base database monitoring system," Computer Applications, vol. 36, no. s1, pp. 237-239, 2016.

[5] Z. Yang, and J. Jiang, "Research on the application of linear function data modeling based on partial differential equations, Journal of Hubei Vocational and Technical College of Industry, vol. 24, no. 5, pp. 104-108, 2011.

[6] Y. Bai, Q. Zhang, and Y. Shang, "The slice similarity anisotropic diffusion model of coupled impact filters," Computer Engineering, vol. 43, no. 3, pp. 271-276, 2017.

[7] H. Zhou, Y. Xue, and Z. Liu, "Measurement and analysis of factors influencing the maintenance cost of UHV substation equipment," Electric Power Construction, vol. 39, no. 1, pp. 19-29, 2018.

[8] X. Zheng, L. Wang, and K. Weng, "Dynamic characteristics analysis of tubular turbine based on bidirectional fluid-solid coupling," Transactions of the Chinese Society of Agricultural Engineering, vol. 32, no. 4, pp. 78-83, 2016.

[9] Y. Wang, P. Zeng, and Y. Zhang, "The maintenance semantic measure of WIA-PA wireless network of smart grid equipment," Journal of Beijing University of Posts and Telecommunications, vol. 38, no. 2, pp. 27-32, 2015.

[10] H. Li, Q. He, and H. Peng, "Application of the nearest neighbor method in annual runoff prediction based on coupling similarity index," Advances in Water Science, vol. 26, no. 2, pp. 161-168, 2015.

[11] Y. Guo, W. Cai, and L. Go, "Telecom intelligent operation system based on big data grid," Telecom Science, vol. 34, no. 6, pp. 159-167, 2018.

[12] Y. Dong, D. Bi, and L. He, "Single image fast defogging algorithm based on two-way coupling," Computer Engineering, vol. 43, no. 10, pp. 246-252, 2017.

[13]C. Ning, and B. Yu, "Constant-strength ductility demand spectral considering degradation, pinching and bidirectional coupling effect," Journal of Vibration and Shock, vol. 36, no. 11, pp. 57-64, 2017.

[14]P. X. Jiang, "A thermo-hydro-mechanics bidirectional coupling mathematical model for drying of biological porous medium," Drying Technology, vol. 33, no. 4, pp. 420-428, 2015.
[15] B. Li, and X. Li, "Study on the effects of microcirculation load based on numerical simulation of aortic flow-solid bidirectional coupling," Medical Biomechanics, vol. 31, no. 5, pp. 426-430, 2016.

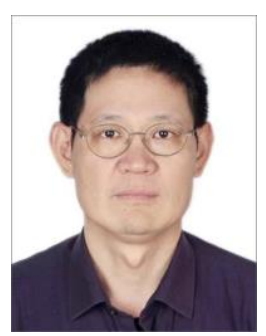

Hongwen Xia, male, was born in November, 1968. His title is professor. He graduated from Hubei Normal University in 1991, major in physics, with a baccalaureate. He graduated from Central China Normal University in 2005, major in educational technology, with a master's degree. He graduated from Huazhong University of Science and Technology in 2006, major in Higher education, with a doctorate. Now he is working in College of Teacher Education, Zhejiang Normal University. He mainly engages in the research of Design and development of intelligent education environment. So far, he has published more than 40 academic papers, presided over or participated in 4 national projects and 7 provincial and ministerial projects. It has successively won the second prize of national teaching achievement and the second prize of excellent achievement of philosophy and Social Sciences in Zhejiang Province.

\section{Author Contribution}

Hongwen Xia: In order to solve this problem, this paper proposes a method to build an enterprise level database operation and maintenance measurement platform based on bidirectional coupling algorithm. The experimental results show that the operational measurement platform built by this method has high throughput, low memory occupancy rate, high measurement accuracy and good user experience. He made all his contributions to the author of this article.

\section{Creative Commons Attribution License 4.0 (Attribution 4.0 International, CC BY 4.0)}

This article is published under the terms of the Creative Commons Attribution License 4.0 https://creativecommons.org/licenses/by/4.0/deed.en US 